ACCEPTED MANUSCRIPT

\title{
Transmission XMCD-PEEM imaging of an engineered vertical FEBID cobalt nanowire with a domain wall
}

To cite this article before publication: Alexis Wartelle et al 2017 Nanotechnology in press https://doi.org/10.1088/1361-6528/aa9eff

\section{Manuscript version: Accepted Manuscript}

Accepted Manuscript is "the version of the article accepted for publication including all changes made as a result of the peer review process, and which may also include the addition to the article by IOP Publishing of a header, an article ID, a cover sheet and/or an 'Accepted

Manuscript' watermark, but excluding any other editing, typesetting or other changes made by IOP Publishing and/or its licensors"

This Accepted Manuscript is @ 2017 IOP Publishing Ltd.

During the embargo period (the 12 month period from the publication of the Version of Record of this article), the Accepted Manuscript is fully protected by copyright and cannot be reused or reposted elsewhere.

As the Version of Record of this article is going to be / has been published on a subscription basis, this Accepted Manuscript is available for reuse under a CC BY-NC-ND 3.0 licence after the 12 month embargo period.

After the embargo period, everyone is permitted to use copy and redistribute this article for non-commercial purposes only, provided that they adhere to all the terms of the licence https://creativecommons.org/licences/by-nc-nd/3.0

Although reasonable endeavours have been taken to obtain all necessary permissions from third parties to include their copyrighted content within this article, their full citation and copyright line may not be present in this Accepted Manuscript version. Before using any content from this article, please refer to the Version of Record on IOPscience once published for full citation and copyright details, as permissions will likely be required. All third party content is fully copyright protected, unless specifically stated otherwise in the figure caption in the Version of Record.

View the article online for updates and enhancements. 


\title{
Transmission XMCD-PEEM imaging of an engineered vertical FEBID cobalt nanowire with a domain wall
}

A Wartelle ${ }^{1}$, J Pablo-Navarro ${ }^{2}$, M Staňo $^{1}$, S Bochmann $^{3}, \mathbf{S}$ Pairis $^{1}, \mathrm{M}$ Rioult $^{4}, \mathbf{C}$ Thirion ${ }^{1}, \mathbf{R}$ Belkhou ${ }^{4}, \mathbf{J}$ M de Teresa ${ }^{2,5,6}, \mathbf{C}$ Magén $^{2,6,7}$ and O Fruchart ${ }^{8}$

${ }^{1}$ Univ. Grenoble Alpes, CNRS, NEEL, F-38000 Grenoble, France

2 Laboratorio de Microscopías Avanzadas (LMA), Instituto de Nanociencia de Aragón (INA), Universidad de Zaragoza, E-50018 Zaragoza, Spain

${ }^{3}$ Friedrich-Alexander-Universität, Erlangen, Germany

${ }^{4}$ Synchrotron-SOLEIL, Saint-Aubin, BP48, F91192 Gif sur Yvette Cedex, France

5 Instituto de Ciencia de Materiales de Aragón (ICMA), Universidad de Zaragoza-CSIC, E-50009 Zaragoza, Spain

${ }^{6}$ Departamento de Física de la Materia Condensada, Universidad de Zaragoza, E-50009 Zaragoza, Spain

${ }^{7}$ Fundación ARAID, 50018 Zaragoza, Spain

${ }^{8}$ Univ. Grenoble Alpes, CNRS, CEA, Grenoble INP $\ddagger$, INAC, SPINTEC, F-38000 Grenoble, France

E-mail: alexis.wartelleaneel. cnrs.fr

\begin{abstract}
Using Focused Electron-Beam-Induced Deposition (FEBID), we fabricate a vertical, platinum-coated cobalt nanowire with a controlled three-dimensional structure. The latter is engineered to feature bends along the height: these are used as pinning sites for domain walls, which are obtained at remanence after saturation of the nanostructure in a horizontally applied magnetic field. The presence of domain walls is investigated using Xray Magnetic Circular Dichroism (XMCD) coupled to PhotoEmission Electron Microscopy (PEEM). The vertical geometry of our sample combined with the low incidence of the Xray beam produce an extended wire shadow which we use to recover the wire's magnetic configuration. In this transmission configuration, the whole sample volume is probed, thus circumventing the limitation of PEEM to surfaces. This article reports on the first study of magnetic nanostructures standing perpendicular to the substrate with XMCD-PEEM. The use of this technique in shadow mode enabled us to confirm the presence of a domain wall (DW) without direct imaging of the nanowire.
\end{abstract}

Keywords: FEBID, ferromagnetic nanowires, three-dimensional nanostructures, XMCDPEEM, transmission microscopy, core-shell structures, domain walls

PACS numbers: 75.75.Cd, 78.20.Ls, 79.60.Jv, 75.75.Fk

Submitted to: Nanotechnology

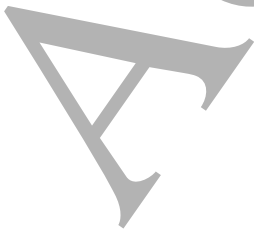




\section{Introduction}

As nanofabrication techniques and magnetic imaging methods evolve, novel magnetic nanostructures not limited to two dimensions are increasingly investigated $[1,2]$. The use of bottom-up approaches such as electrodeposition into nanoporous templates [3, 4], strain engineering [5] and self-assembly induced by the decomposition of a thinfilm matrix [6] has enabled the production of magnetic micro- or nanoparticles with a large variety of geometries and materials. However, the samples are often limited to straight cylindrical or tubular shapes $[7,8,9,10$, 11], a restriction that can be lifted with FEBID. This technique allows one to deposit inside a Scanning Electron Microscope (SEM) the desired material on the electron beam spot with a lateral size below $100 \mathrm{~nm}[12,13]$ with the possibility to move that spot during growth as well as rotate or tilt the substrate. As a result, it is possible to grow structures in two or three dimensions $[14,15,16]$ with the few limitations arising from the directional character of the technique. Moreover, tuning the purity of the deposited metal either during the deposition process $[17,18]$ or postfabrication [19] allows one to further tailor the structure properties. In the case of magnetic nanostructures, cobalt is most often used [12], and the deposits are polycrystalline with no texture and grain size below $10 \mathrm{~nm}[20,21]$.

We investigate here a Co structure grown in the shape of a near-cylindrical nanowire perpendicular to the silicon wafer substrate and coated with FEBID-grown $\mathrm{Pt}$ [22], as explained in the following section. It was fabricated with several well-defined bends as can be seen in figure 1.a) and $b$ ), featuring the nanostructure whose magnetic state which will be the focus in the following. The purpose of its geometry is to obtain DWs at remanence as sketched in figure 1.c), after having saturated the sample with a horizontal magnetic field (i.e. in the plane of the substrate) aligned with the bends. Such a method was demonstrated in soft cylindrical nanowires [23] and is also applicable in our case considering the soft nature of our magnetic material.

Neither conventional magneto-optical nor magnetic force microscopy are suitable for the study of such vertical samples, contrary to shadow XMCD-PEEM $[1,24,25,26$, 27]. We choose this most relevant technique since it probes magnetization in the sample volume with the added value of element specificity, as opposed to electron holography [28] which is sensitive to the total magnetic induction.

With respect to experiments involving nanostructures lying on the substrate $[24,25,29]$, the vertical geometry provides access to a larger proportion of the shadow. The goal is to assess the presence of DWs thanks to the latter. Furthermore, the spectroscopic capabilities of the technique allow us to probe the metallic nature of the deposited material in the volume thanks to the transmission of X-rays through the sample. To our knowledge, this is the first study of such a vertical, high-aspect ratio nanostructure with shadow XMCD-PEEM, in contrast with previous studies on three-dimensional samples of diverse shapes $[30,31]$ and orientations [27]. We demonstrate that retrieving the magnetic state of vertical samples is possible without the complex analysis required in (ptychographic) tomography $[32,33]$ and without the need for a transparent substrate [32] or large spatial separation between substrate surface and actual sample for visibility [33]. By contrast, in PEEM, the substrate is key in the imaging with few requirements other than being conductive and flat, thus facilitating the sample preparation. Our investigation demonstrates the technical feasibility of imaging engineered vertical ferromagnetic nanowires with shadow XMCD-PEEM and paves the way for quantitative studies and the investigation of more complex vertical structures, such as core-shell nanowires with different ferromagnetic materials, elaborate 3D architectures [34] that may be micromanipulated onto the imaging substrate [33] if need be, or nanostructures grown into porous templates [9].

\section{Sample fabrication}

The three-dimensional (3D) core-shell structure was grown in the commercial Helios Nanolab 650 Dual Beam system using the Schottky field-emission gun (S-FEG) electron column and the gas injector systems (GIS) for Co and $\mathrm{Pt}$ depositions using $\mathrm{Co}_{2}(\mathrm{CO})_{8}$ and $\mathrm{CH}_{3} \mathrm{CpPt}\left(\mathrm{CH}_{3}\right)_{3}$ precursor gases, respectively. The substrate was a $\mathrm{Si}$ wafer with native oxide. Firstly, the Co nanowire core was grown with a $5 \mathrm{kV}$ electron voltage and a $100 \mathrm{pA}$ beam current at a gas pressure of $3.33 \times 10^{-5} \mathrm{mbar}$ (base pressure of $4.73 \times 10^{-6} \mathrm{mbar}$ ). The stage stands still with zero degree tilt throughout the Co deposition. The pattern is composed of 77 points separated $14 \mathrm{~nm}$ in a straight line parallel to the flat end of the Co GIS, keeping constant the precursor molecules flux over the whole deposition. The nanowire is made of seven segments, each one having its particular growth strategy. Vertical segments are obtained by scanning a single pattern point while the electron beam stands still; by contrast, bent segments are fabricated by scanning a sequence of points while shifting the electron beam position. For a fixed total horizontal shift during the sequence, the segment's angle with respect to the substrate 


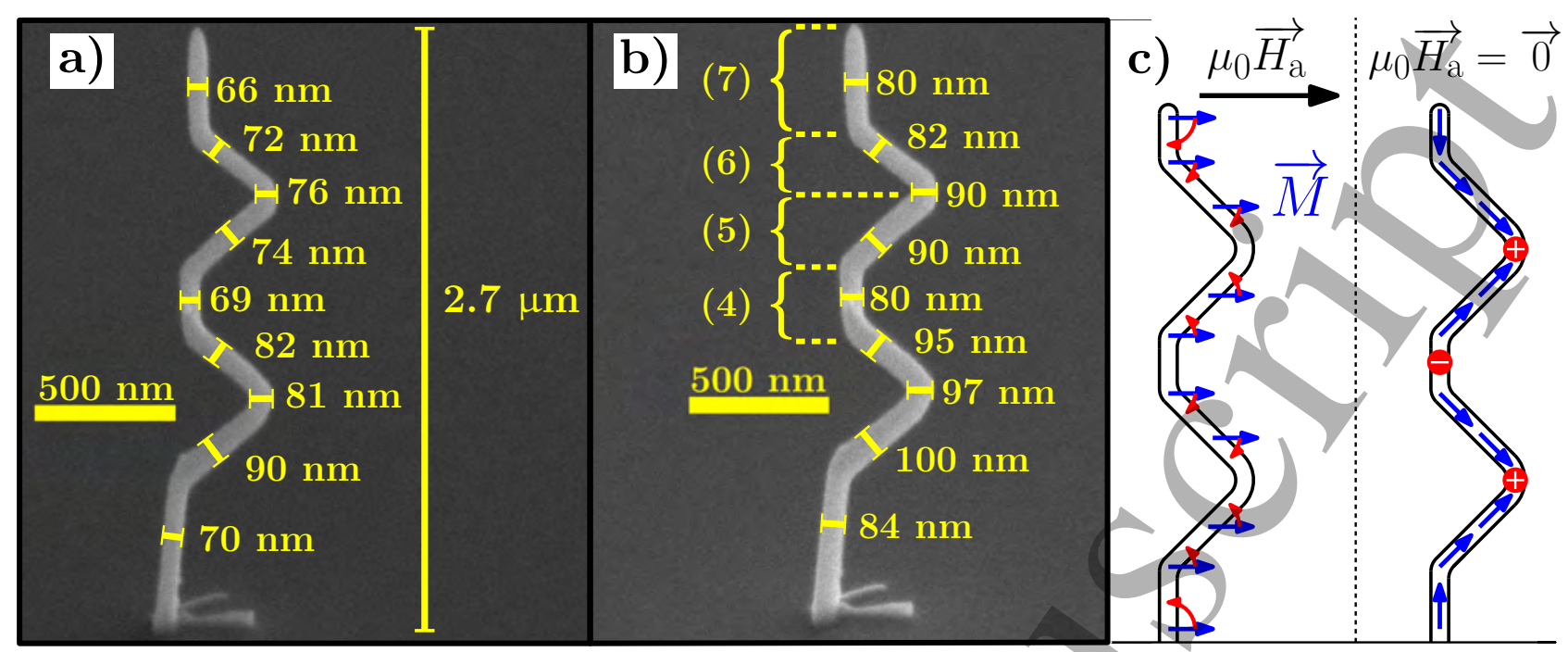

Figure 1. SEM views at $52^{\circ}$ stage tilt (with diameter and tilt-corrected height indications) of a) the as-grown Co FEBID nanowire and b) the structure after FEBID Pt coating. The four top segments (4), (5), (6) and (7) will be discussed further in the section pertaining to the magnetic state of the nanowire. c) Scheme for application of the in-plane magnetic field leading to three DWs of alternating polarity (red-rimmed black dots) at remanence.

depends on the number of points. The higher that number, the shorter the distance between two subsequent points: therefore the overlap between them will be higher and the angle with respect to the substrate will increase. In our case, each bent segment corresponds to 18 pattern points with a scanning time of $97.2 \mathrm{~ms}$. To form the $90^{\circ}$ bends, the joint between the bent segments is fabricated by a single point scanned for $581.2 \mathrm{~ms}$. Then, the bend is completed by reversing the electron beam shift direction.

The first segment of the nanowire's cobalt core was grown by depositing on the first point ( $2903.8 \mathrm{~ms}$ at the same position). The second segment and the third one were then grown as described above, taking into account that the points of the latter are exactly over those of the former but gone through in the reverse direction, thus forming the first bend of the nanostructure. The forth segment growth was completed within $1549.1 \mathrm{~ms}$ scanning of the same point. Then, the fifth and the sixth segments, as well as the joint between them were carried out just as the first bend. The top segment was fabricated scanning the last point during $2419.8 \mathrm{~ms}$.

The Pt shell was grown immediately after the ferromagnetic core $(\sim 65-70 \%$ at. Co $)$ in order to avoid its oxidation, following the process described in a previous work [22]. The experimental conditions were $5 \mathrm{kV}$ electron voltage and $100 \mathrm{pA}$ beam current at $2.40 \times 10^{-5} \mathrm{mbar}$. First, tilting the stage $52^{\circ}$ the nanostructure is seen as in figure 1.a) and a polygonal pattern of Pt deposition ( $2 \mathrm{~s})$ is set following the shape of the nanowire core viewed from this perspective. Then, to complete the coating, the stage is rotated $180^{\circ}$ and a new deposit is carried out in the same way just on the opposite side. Pt deposition increases the nanowire diameter by approximately $13 \mathrm{~nm}$, as seen in figure 1.b), just enough to prevent Co oxidation.
Investigations of the microstructure of core-shell nanowires prepared in the same way [22] (except for the geometry with bends) have shown that the cobalt core is nanocrystalline with no texture, thus ensuring the magnetically soft nature of our material.

\section{XMCD-PEEM imaging and characterization}

The XMCD-PEEM experiments have been performed on the XPEEM branch of the HERMES beamline (Synchrotron SOLEIL - France) [35]. Several silicon wafers featuring rows of nanostructures similar to the aforementioned sample were brought for investigation at the $\mathrm{Co}_{3}$ edge, which is associated with large magnetic dichroism. Despite the apparent fragility of the nanowires arising from their high aspect ratio, the majority of those which we tried to image on site was still standing. Aside from shocks during transportation, the structures may have been damaged during sample mounting and also by the imaging technique itself. Indeed, as the samples are subjected to a high voltage of $20 \mathrm{kV}$ (with respect to the microscope objective), a large field emission of electrons due to the tip of the structures is generated. From the thermal perspective, the $\mathrm{Si}$ substrate through the narrow base of the structure is the only heat sink in the vacuum environment. However, different and previous experiments in this microscope [36] show that the resonant absorption of X-rays on even a full-scale thin film sample results in temperature increases less than $10 \mathrm{~K}$. The current associated to the field emission has not been quantified, yet similar nanowires have been observed [15] to strongly distort or even to be destroyed when subjected to large temperature increases. This is not the case, as indicated by figure 3 . As far as potential microstructural changes 


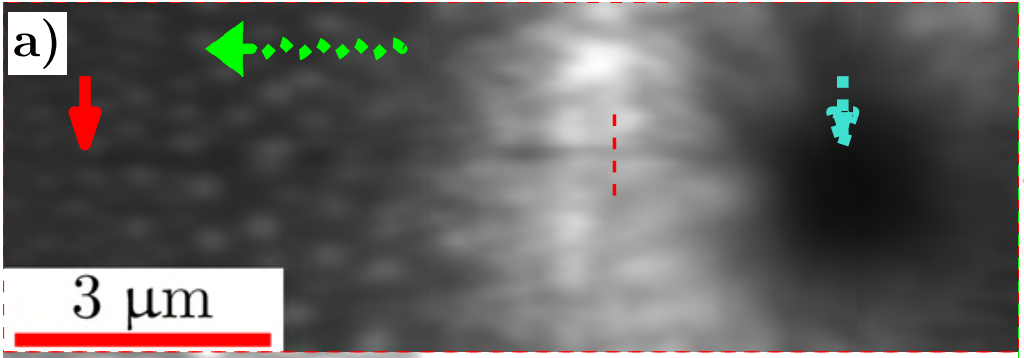

are concerned, we could not obtain conclusive data because of sample breaking during post-experiment transportation, yet, we have grounds to neglect them. In the following paragraph, we will show that Joule heating due to the fieldemission-induced current is negligible.

The current flowing from the whole sample to the microscope is on the order of a few dozen nA at most during experiments. Neglecting the presence of the dozen or so other nanowires on the substrate, it is safe to state that the maximum current through our nanowire was at most $100 \mathrm{nA}$. We assume our wire's resistivity to be comparable to that measured by Begun and coworkers on similar (but uncoated) FEBID Co depositions [37], i.e. a few $\mu \Omega \mathrm{m}$. Since the structure stands in ultra-high vacuum, the corresponding Joule heating can only be evacuated via the silicon wafer, the latter being thermalized at room temperature from the sides and bottom surface. As for the nanowire's thermal conductivity, we use a temperature-dependent composition- and geometryweighted average of the thermal conductivities of bulk $\mathrm{Co}, \mathrm{Pt}$, and amorphous carbon $[38,39]$. We also assume our silicon substrate's thermal conductivity to be that of bulk $\mathrm{Si}$. If we now solve numerically the stationary one-dimensional heat diffusion problem (we simplify the problem by considering a $1 \mathrm{D}$ straight wire), we obtain a maximum temperature increase less than one kelvin. In other words: starting from reasonable material parameters, even a clearly overestimated field-emission current leads to a negligible Joule heating. Therefore, the sample's

nanocrystalline and magnetically soft nature must have

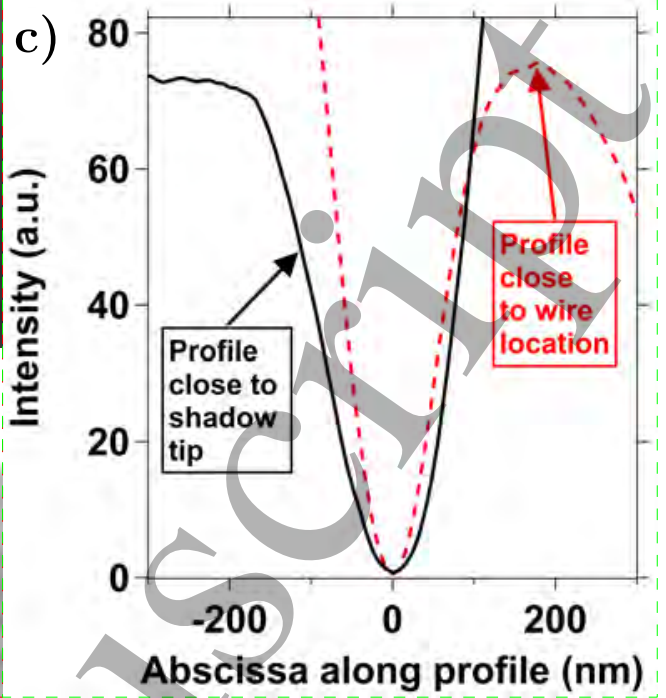

differ only in the linear gray scale: the range is 4.3 times Figure 2. a) and b): PEEM images of the surroundings of the nanostructure. The two images differ only in the linear gray scales
smaller on b) with a slightly higher minimum with respect to a), so that the maximum extent of the nanostructure's shadow (whose horizontal direction is that of the X-ray beam, illustrated with the wavy green arrow) is better seen. On both images, the latter's tip is indicated by the red arrow, and the wire location by the light blue arrow. The red dashed and black full lines indicate the position of intensity profiles extracted from the images. c) Profiles demonstrates that the shadow is wider at its tip.

been preserved during the experiment.

The field emission is however of practical use in the search for standing structures as it creates wide, strongly contrasted features directly at the location of the nanowires. It corresponds to the dark area to the right of the PEEM images in figure 2.a) and b). These images are of the sample shown in figure 1.a) and b), sole nanowire that was investigated in this work. The low intensity is expected; indeed, the current associated to the field-emission does not contribute to the image because it is rejected by the energy slits of the microscope. In addition, this positive current flowing downwards i.e. along the structure towards its foot, results in a negative charge density close to the wire's tip, as well as an Oersted-type magnetic field. Both deflect the photoelectrons from the nanostructure, which would build up the direct image on the detector. Since these electrons are selected with a contrast aperture rejecting photoelectrons with a wave-vector different from that of the photoelectrons from the substrate, only a vanishing signal is detected in this area. Furthermore, distortions due to the strong and inhomogeneous electric fields at the nanowire tip must be taken into account. As a result, no direct imaging of the structure could be performed and hence no XMCDPEEM imaging of the nanowire's surface magnetization. However, when visible, its shadow (resulting from resonant absorption of X-rays) contains enough information [1] to deduce the structure's magnetic state, as will be shown later.

Due to the incidence angle of $18^{\circ}$, the total shadow length is increased with respect to the structure height by a factor $1 / \sin \left(18^{\circ}\right) \simeq 3.2$. Therefore, one way to retrieve 
the nanowire's height is to measure the extent of its shadow: it can easily be traced on both sides since the center of the very dark area on the PEEM images corresponds to the location of the structure. From the images, the shadow length is about $8.1 \pm 0.3 \mu \mathrm{m}$, which results in a height of $2.5 \pm 0.1 \mu \mathrm{m}$, while SEM measurements performed before shipping of the samples indicated $2.7 \mu \mathrm{m}$. SEM investigation after the synchrotron experiments indicated that the nanostructure was bent with respect to its initial state, as is illustrated in figure 3. The measured height was $2.3 \pm 0.1 \mu \mathrm{m}$. The agreement between both height estimates confirms that some plastic deformations were induced, possibly during observation. Such deformations have previously been observed for such structures simply upon aging. Since in this case the sample was also exposed to large electric fields, such differences with respect to its initial state are not surprising.

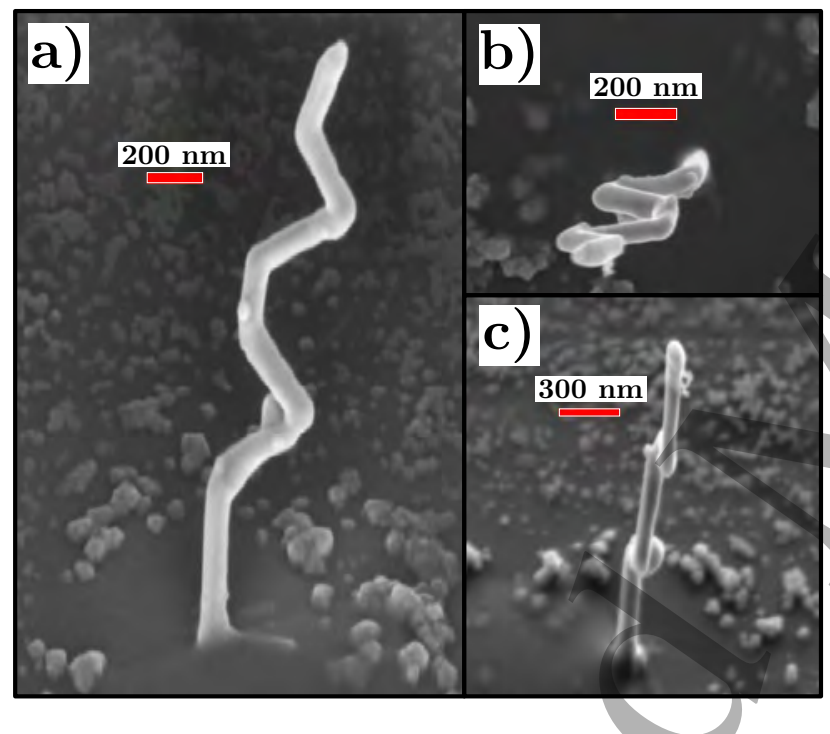

Figure 3. SEM observations of the nanowire after the XMCD-PEEM experiments a) at $45^{\circ}$ tilt, view perpendicular to the bends b) from the top, untilted stage c) along the bends with $45^{\circ}$ tilted stage.

As was shown in the case of three-dimensional samples (with dimensions significantly exceeding the mean free path of photoelectrons, $\sim 10 \mathrm{~nm}$ ) lying on a conductive substrate $[1,24,25,40]$, XMCD-PEEM can be used in transmission or so-called shadow mode. With respect to directly illuminated parts of the substrate, the shadow regions display a reduced intensity according to the magnetization-dependent absorption through the structure. Since circular dichroism is integrated along the X-ray path through the structure, which may be nonuniform in thickness and/or magnetization configuration, not all the three-dimensional information from the volume is accessible from this projection onto the substrate. Nevertheless, it was shown that the post-processing of simulated micromagnetic configurations allows one to simulate the shadow XMCD-PEEM contrast of a given object with qualitative [25] and even quantitative agreement
[26]. This capability is crucial in the case of strongly inhomogeneous textures such as DWs where the shadow contrast pattern may be non-trivial.

In our case, the complexity is reduced as we are interested in assessing the presence of DWs, not their internal structure. Furthermore, the observation of our sample in transmission also allows us to check that in the bulk, the sample is weakly if at all oxidized. Similarly to Electron Energy Loss Spectroscopy (EELS) and with a comparable or somewhat lesser energy resolution [35, 41], the technique enables to study the characteristic peak of the metal(s) of interest, in our case cobalt, and determine if the bulk material is rather metallic or oxidized based on the absence or presence of a multiplet structure.

\subsection{Imaging and spectroscopic characterization}

Considering the $\mathrm{Co}_{2}(\mathrm{CO})_{8}$ precursor, the bright dots that can be seen around the nanowire consist of cobalt, oxygen and carbon. Their thickness is on the order of $100 \mathrm{~nm}$ or less, as observed with SEM. Establishing correspondence between PEEM imaging at the Co $\mathrm{L}_{3}$ edge and SEM imaging is not straightforward because of the interplay between topography and local composition. Cobalt as element is revealed by the resonant imaging at an absorption edge of this element, with the wire shadow (X-rays absorbed by the nanostructure) and the strong halo intensity as signatures. On the other hand, the presence of oxide and/or carbide around the nanowire may be inferred by X-ray Absorption Spectroscopy (XAS) data on the inset of figure 4: the $\mathrm{Co}_{3}$ peak displays a multiplet structure incompatible with metallic cobalt [42], whereas a similar shape was reported for $\mathrm{CoO}$ [43] and cobalt carbide [44]. It appears that the dots are probably very similar to one another given the degree of similarity between the spectrum of an ensemble of them (red curve in the inset of figure 4) and that of one given dot (thick blue curve). This peak structure is much more pronounced (as shown by the green curve on the inset of figure 4) closer to the structure, where there seems to be an almost continuous layer. This halo is known [45] to originate from secondary electrons created by back-scattered electrons. The spectra on the inset of figure 4 were rescaled so that the pre-edge intensities match, in order to allow for comparison.

On the other hand, the shadow features spectral information about the volume of the nanowire. Figure 4 illustrates XAS data extracted from the shadow of the nanostructure after subtracting the background averaged over an area of a few square microns close to the shadow's location. The red (blue) curve displays the XAS intensity for the circularly right (left) X-ray polarization. The data is noisy because of the strong background: the signal-tonoise ratio is on the order of a few percents only. Yet, the multiplet structure seems less pronounced as far as noise allows us to tell despite the fact that the outer rim of the cobalt rod (where the purity is decreasing) is included 


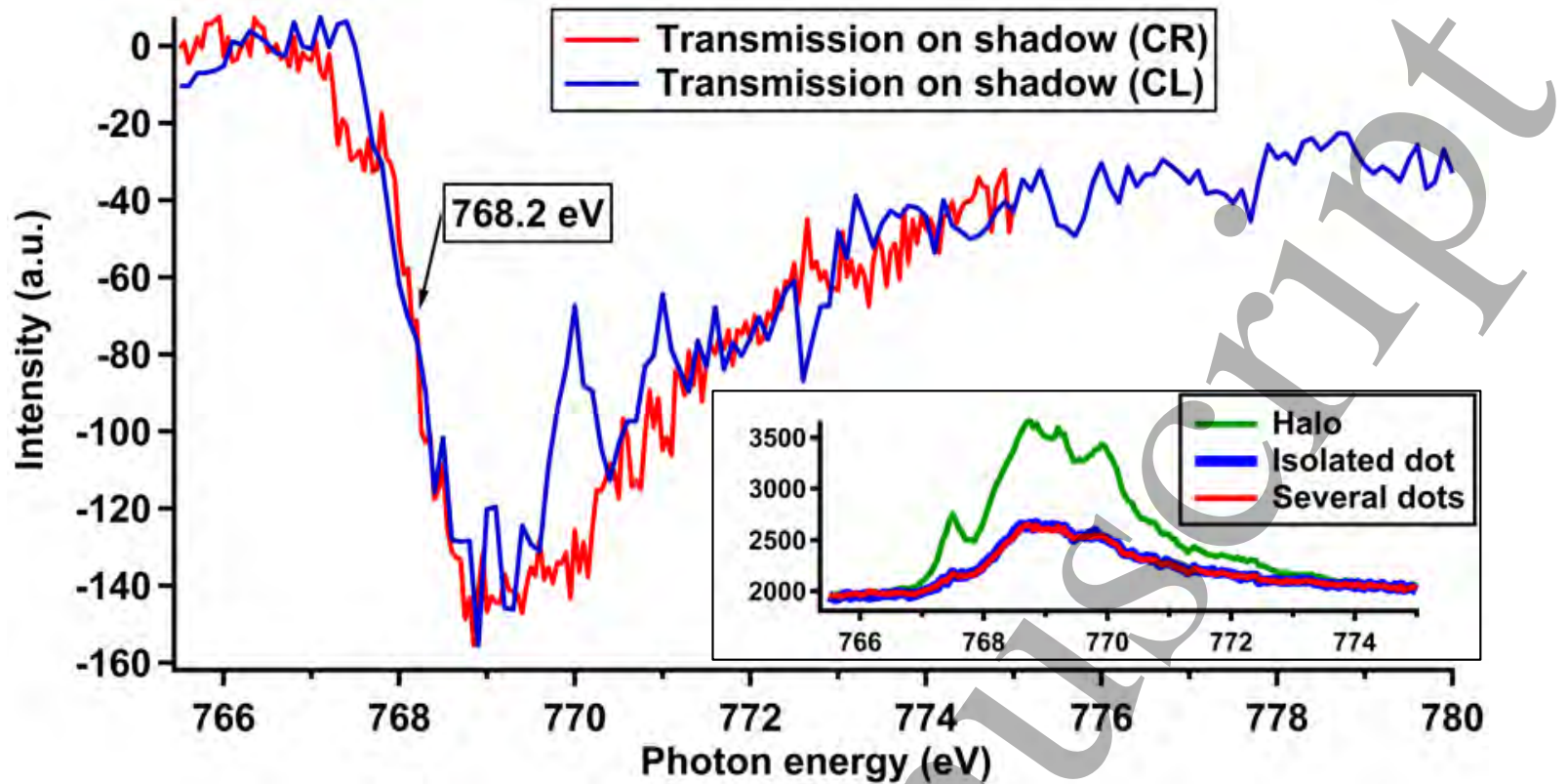

Figure 4. XAS on the structure's shadow, featuring the decrease in transmission as the photon energy is sweeped through the Co $\mathrm{L}_{3}$ edge. The blue (red) curve corresponds to circularly left (right) polarized light. The target working energy of $768.20 \mathrm{eV}$ is indicated. The inset displays XAS on the background where the $\mathrm{CoO}$ dots are more isolated (red and thick blue curves), and on the halo close to the nanostructure (green curve).

in the absorption spectra and thus decreases the averaged Co metallicity. We have therefore an indication of more metallic material in the volume of the sample, which was protected from oxidation by the Pt coating [22]. The arrow indicates the target working energy $768.20 \mathrm{eV}$ taken as $\mathrm{Co}_{3}$. Throughout this report, only the target photon energies are indicated for consistency with the energy steps $(0.05 \mathrm{eV}$ i.e. below the instrumental energy resolution of $\delta E=0.1 \mathrm{eV}$ ) used to record absorption spectra. Since the latter were acquired on a part of the shadow with uniform XMCD contrast [see white segment (4) in figure 5.b)], the difference in intensity should reflect this contrast. This difference is however very small (possibly below noise), nevertheless, this working energy yielded the best quality for XMCD-PEEM images. Imaging was also performed at the top of the peak, at $769.10 \mathrm{eV}$, as well as $769.85 \mathrm{eV}$ where the difference between the spectra is the largest, but the contrast amplitude at these energies is even lower than at $768.20 \mathrm{eV}$. This behaviour is by itself an indication that non-metallic components are not completely negligible: indeed, circular dichroism in nonoxidized metals is strongest at the energy corresponding to the maximum of absorption [42].

\subsection{Magnetic state of the wire}

As was indicated in the introduction, before mounting the sample into the microscope, a horizontal field was applied in the direction of the bends with an electromagnet so as to saturate the whole nanowire. The field strength was $\mu_{0} H_{\mathrm{a}}=0.73 \mathrm{~T}$. Upon decreasing the strength of the field to zero, we expect three DWs to be nucleated as shown in figure 1.c). This process relies on the soft nature of the material and has been successfully implemented by $\mathrm{Da} \mathrm{Col}$ and coworkers [23] in curved permalloy nanowires. The pinning of domain walls due to bends has been reported in several experimental [46, 47, 48, 49] and theoretical [50] works, the experiments showing an increase in pinning both with the curvature and the angle of the bends [48, 49].

In order to understand the DW nucleation process, let us first consider a cylindrical geometry and a soft material in a Stoner-Wohlfarth approach. A field $H_{\text {sat }}$ equal to half the spontaneous magnetization should be sufficient to reach saturation, if applied at $90^{\circ}$. In the case of pure bulk Co, one would have $\mu_{0} H_{\mathrm{sat}}^{(0)}=\mu_{0} M_{\mathrm{s}, \mathrm{Co}} / 2=0.89 \mathrm{~T}$. This is higher than the applied field $\mu_{0} H_{\mathrm{a}}=0.73 \mathrm{~T}$.

In our case, we must heed the angle of about $45^{\circ}$ of the structure's segments (2), (3), (5), (6) with respect to the field. However, we need only to be above their switching field so that magnetization is mainly along the applied field, and features DWs at remanence. This reduces the field (still in a Stoner-Wohlfarth approach) by a factor 2 and therefore the induction needed is: $\mu_{0} H_{\mathrm{sw}}=0.45 \mathrm{~T}<\mu_{0} H_{\mathrm{a}}$. Thus, assuming that the magnetic field is exactly at $45^{\circ}$ with the bent segments, we are confident to have reached switching in the relevant wire segments without needing to invoke a reduced spontaneous magnetization (with respect to pure bulk cobalt). Moreover, based on the StonerWohlfarth astroid, the difference $H_{\mathrm{a}}-H_{\text {sw }}$ provides us with a margin on the angle at which the field is actually applied. Indeed, its amplitude is large enough to enforce switching in the bent segments even if the field is not perfectly 
horizontal: we estimate the margin to be about $\pm 40^{\circ}$, which is much larger than the error that can be expected in our experiment. As a result, we no longer need to assume a perfect alignment of our magnetic field. It should be noted that in the case of the vertical segment (4), any slight tilt of the structure results in a non-zero projection of segment (4) along the field $\overrightarrow{H_{a}}$. Therefore, the DW denoted as a redrimmed black dot in figure 1.c) with a minus sign may move towards a neighbouring bend, from (3) to (4) or from (4) to (5), and be pinned there.

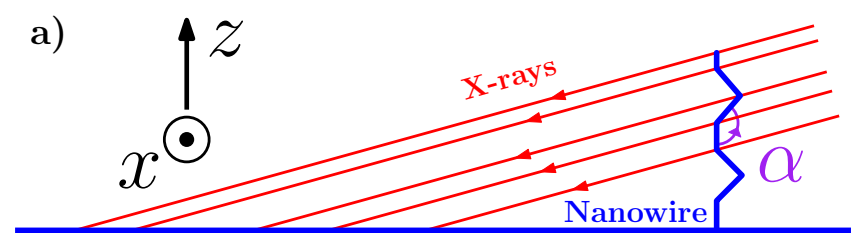

(7) (6) (5) (4)
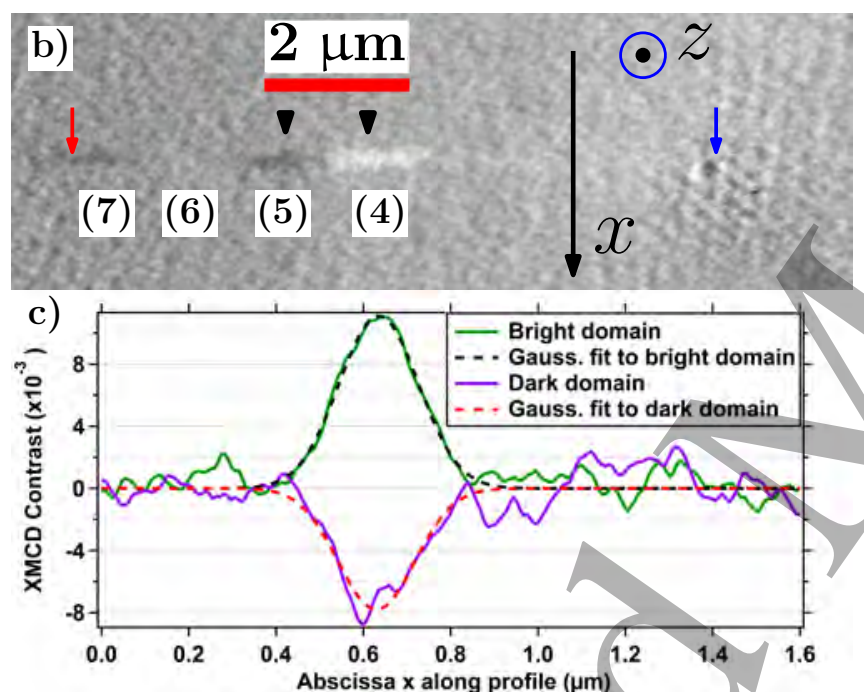

Figure 5. a) True-to-scale schematic representation of the X-ray beam falling onto the nanowire; the scale length is as in figure 5.b). The rays passing through junctions between segments delimit the shadow sections corresponding to segments (4) through (7). b) Shadow XMCD-PEEM image of the nanowire with red arrow indicating the tip of its shadow and dark blue arrow indicating the approximate nanowire position. The domains with uniform contrast correspond to the segments (4), (5) and (7) from figure 1.b). The two black markers above segments (4) and (5) indicate the lateral positions of the contrast profiles in figure 5.c). The transition region between segments (4) and (5) is where a DW was pinned. c) Contrast profiles across the shadow in domains (5) (in purple) and (4) (green), along with their best gaussian fits in dashed lines. The linear gray scale goes from $-2.5 \%$ to $+2.5 \%$. To the right, the noise is slightly stronger where the structure stands because of the field emission.

A schematic but true-to-scale view of the X-ray beam falling onto the structure is featured in figure 5.a): it illustrates how the subsequent nanowire segments (4) through (7) are projected onto the substrate to form their respective shadow sections. One should note that the nanostructure's possible tilt is not featured. Correspondingly, the XMCD-PEEM image of the structure's shadow shown in figure 5.b), with a red (resp. blue) arrow indicating its tip (resp. the nanowire's position) reproduces these sections, which are vertically aligned with their counterparts in figure 5.a). The structure itself is located on the right on the image, where the noise is stronger due to the field emission causing a strong local reduction of intensity. Away from the shadow, the contrast is zero, indicating that the dots and the halo are not ferromagnetic, as is suggested by the work of Fernández-Pacheco et al. [15]. The shadow itself features three identifiable domains: a faint dark domain at the tip corresponding to segment (7), and further away from the latter another dark domain in segment (5) followed by a bright one in segment (4). First of all, the position of the transition from (4) to (5), about $5.5 \pm 0.3 \mu \mathrm{m}$ away from the structure, is consistent with its SEM-measured height [see figure 1.a)] of $1.5 \pm 0.1 \mu \mathrm{m}$ : $(5.5 \pm 0.3 \mu \mathrm{m}) \times \sin \left(18^{\circ}\right)=1.7 \pm 0.1 \mu \mathrm{m}$. Even though the background emission is strong, the close to $1 \%$ contrast amplitude is clearly above noise in the contrast profiles of figure 5.c). Regarding the magnetic state of the observable parts of the nanowire's shadow, let us first recall that if we name $\varphi$ the angle between the beam's wave vector and magnetization, the XMCD contrast is proportional to $\cos \varphi$. Now, the absence of contrast in segment (6) is due to both the beam's incidence angle ( $\varphi$ closest to $90^{\circ}$ among all visible segments) and the presence of a bright dot in this area, as can be seen in figure 2.a) or b). This dot does not contribute to the dichroic signal because of its non-ferromagnetic nature, but does enhance the background level and therefore reduces the signal-to-noise ratio [26]. Similarly, to the right of segment (4), the halo emission is too strong and no magnetic contrast can be retrieved from this part.
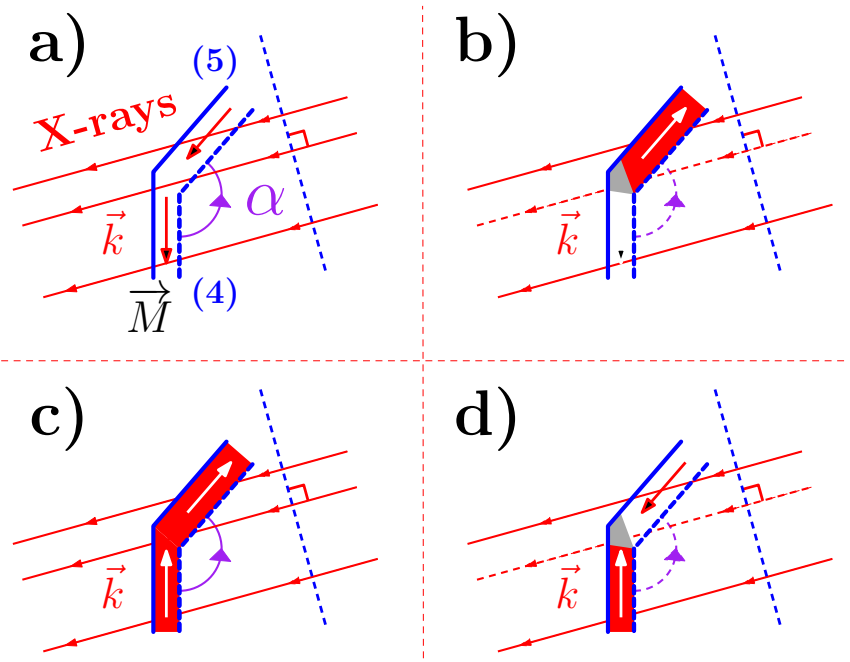

Figure 6. Schematic view of the four possible magnetic configurations [a) through d)] at the bend between segments (4) and (5) (in blue). The Xray beam's wave vector $\vec{k}$ (in red) determines the shadow XMCD-PEEM contrast $\propto \vec{M} \cdot \vec{k}$, here represented inside the wire segments for simplicity. Considering the orientations at play, our experiment corresponds to b), indicating the presence of a domain wall (in gray) in the bend. 
The soft nature of the deposited cobalt, along with the constant contrast sign within segments (7), (5) and (4), suggests that magnetization is uniform inside them. As for the change in contrast sign from segment (5) to segment (4), we must be careful because its interpretation heavily depends on the angle $\alpha$ (see figure 5) between them.

Remembering that the contrast is proportional to $\vec{M} \cdot \vec{k}$, where $\vec{M}$ is magnetization and $\vec{k}$ the X-ray wave vector, we must consider the direction of the segments (4) and (5), since magnetization will be aligned to them. Figure 6 illustrates the four possible scenarios at this bend, taking into account all relative orientations in our experiment. It can be seen that the only situation corresponding to our contrast pattern is that of figure 6.b). The key feature is a change in contrast sign; thanks to the latter, we can conclude that magnetization reverses from segment (5) to segment (4): in other words, a DW is pinned in this bend. Thus, we confirm the suitability of our nanowire geometry and material for a controlled creation of DWs.

We tried to compute the expected transversal contrast profile across the structure's shadow and compare its shape with the curves in figure 5.b). The agreement between the data and a gaussian profile $f$ defined by $f(x)=$ $A \exp \left[-(x / \Delta)^{2}\right]$ is very good, with fitting results $\Delta^{(\exp )} \simeq$ $120 \mathrm{~nm}$ in both cases. It is noteworthy that the actual diameter of the Co rods is about $70 \mathrm{~nm}$. At first sight, this agreement is surprising because the theoretical profile should be of the form:

$C(x)=A \tanh \left(\kappa \sqrt{1-\frac{x^{2}}{d^{2}}}\right)$

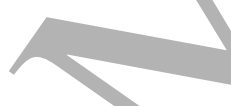

(1)

where $\kappa$ is proportional to the difference $\delta \mu$ in linear absorption coefficients of X-rays in Co (for magnetization parallel and anti-parallel to the beam at the $\mathrm{Co} \mathrm{L}_{3}$ edge) and the travelled distance through the structure. Here, $d$ is the diameter of the nanowire. Considering the length scales as well as the order of magnitude of $\delta \mu \simeq$ $0.034 \mathrm{~nm}^{-1}$ [42], one would expect a profile much closer to a square function than to a gaussian since we are in the strong absorption regime (except close to the edges), where circular dichroism should saturate. The origin of this shape is partly wave optics: the nanowire diffracts the incoming beam, which leads to an image onto the substrate with different lateral dimensions. Fringe patterns due to Fresnel diffraction on 3D objects have already been reported [26] in XMCD-PEEM experiments. In our case, we are dealing with Fresnel diffraction as well, and a rough numerical computation of the diffraction pattern (not shown here) leads to a satisfactory agreement in terms of profile shape. However, taking into account the instrumental resolution of ca. $30 \mathrm{~nm}$ to $40 \mathrm{~nm}$ as well as the beam's divergence of ca. $0.008 \mathrm{mrad}$ leads to an estimate of the width $\Delta^{(\text {theo })} \simeq$ $80 \mathrm{~nm}$ to $90 \mathrm{~nm}$, in rather poor agreement with $\Delta^{(\exp )}$.

\section{Mechanical oscillations of the nanowire}

A source of broadening for the profile is vibrations of the vertical nanowire caused by fluctuations in the microscope's high voltage and the field emission process. Since the structure is not entirely metallic, variations in the electric field to which it is subjected would lead to random forces exerted upon its tip. Considering how our integration time for image acquisition is on the order of a second, voltage noise of frequencies above a few hertz would lead to a blurred and wider shadow. This is consistent with what can be observed in figure 2.c): the shadow is wider close to its tip with respect to sections closer to the nanostructure's foot. The widths close to the wire foot and close to the shadow tip can be compared thanks to the normalization of both profiles: the function describing the shadow's intensity dip being the same, if its amplitude at both locations is the same, then the curvature at its minimum directly reflects the function's width. The profile close to the shadow tip (black full line) has a clearly weaker curvature than the profile close to the wire foot (red dashed line), resulting in an apparent width about $44 \%$ higher close to the shadow tip despite the slightly lower wire diameter at the tip [see figure 2.b)]. The widths were estimated from fits to second-order polynomials $f(x)=(x / \Delta)^{2}$ of the profiles, close to the minima. The vibrations account not only for the progressive shadow broadening towards its tip and thus the disagreement between $\Delta^{(\text {theo })}$ and $\Delta^{(\exp )}$, but also the profile shape, the absence of diffraction fringes. Furthermore, they contribute to spreading the shadow contrast over a wider area, thus decreasing its measured extremal values. Due to the vibrations and integration time (20 s), the background does contribute to the intensity in the shadow on each PEEM image. As a result, the obtained XMCD-PEEM images see their dichroic contrast reduced because at the shadow's location, the intensity contains a strong non-magnetic contribution. Regarding the DW, the absence of clear features in the corresponding shadow region is also consistent with them being averaged out because of vibrations. While vibrations prevent access to the internal DW configuration in this first-of-its-kind experiment, the technique has been shown to have enough resolution [25] to recover it. Furthermore, the DW position can readily be determined.

The hypothesis of vibrations would first call for confirmation through imaging with lower integration times. Then, using either magnetic field gradients or more likely piezo actuators, one could set the structure tip into periodic motion; in this frame, proper synchronization and short integration times would allow to acquire images with reduced influence of the vibration while keeping the signal to noise ratio sufficiently high. Care would be needed in the case of a magnetic field so that the latter does not disturb the micromagnetic configuration of the nanowire, but there also is a challenge in producing sufficiently strong field gradients in the microscope environment. On the other 
hand, the piezo actuators would provide a more reliable and purely mechanical source of oscillations, with the added value of higher bandwidth. This may seem like an impediment to recovering the full information from the material, but in this frame, the use of tomographic methods and the inclusion of diffraction in the simulations of shadow XMCD-PEEM contrast patterns should allow the resolution of non-uniform magnetization configurations such as DWs.

There is however a possibility that the nanowire is in a self-oscillating regime due to an electric-field-gradientinduced mechanical instability [51]. In this case, the difficulty is increased as the tip motion occurs at the fundamental vibration mode of the nanowire, which a rough estimate places at a frequency $\nu_{1}$ on the order of tens of megahertz. This order of magnitude is comforted by an ongoing work [52] on Focused Ion-Beam-Induced Deposition of vertical tungsten nanowires. The latter were brought into mechanical oscillation by AC electric fields, and the resonance was at a few megahertz. Since tungsten and cobalt (as well as platinum) have Young's moduli of the same order of magnitude, the dominant effect is the nanowire length.

\section{Conclusion}

In summary, we have fabricated vertical, Pt-coated Co nanowires featuring $90^{\circ}$ bends with FEBID and successfully investigated one such structure using shadow XMCD-PEEM. While we lack statistics as only one object was imaged, we consider that the latter is representative of the ensemble of samples. Indeed, the deposition of vertical metallic FEBID pillars has been shown [53] to be homogeneous, and thanks to our previous work on similar samples [22], we are confident in the deposition's reproducibility.

The bulk material quality was checked by XAS in spite of strong background emission from Co-based dots and a continuous halo surrounding the structure. Such an impediment to observations (the major cause for low contrast in our experiment) could be suppressed by deposition of a thick enough metallic layer to prevent the extraction of photoelectrons from Co in the substrate, say $10 \mathrm{~nm}$ of gold or platinum. Another possibility would be to micromanipulate such samples to a location free of deposition halo, away from their fabrication spot. In this frame, a much larger extent of the structures' shadow should be revealed up to the close vicinity of the investigated structure, and a significantly larger signal-tonoise ratio achieved: the $\sim 1 \%$ contrast amplitude is by far not an upper limit, as was shown in previous works using shadow XMCD-PEEM [1, 25]. Moreover, the Co content and associated magnetization could be increased by postgrowth annealing strategies, thus enhancing the magnetic contrast $[37,54]$.

The majority of the structures that were glimpsed (but not investigated or imaged in detail) in the experiments were still standing, which testifies of their robustness despite their non-conventional geometry. Thanks to the latter and the application of an in-plane field, we were able to conclude from the shadow XMCD-PEEM contrast that at least one DW was present in the structure. Unfortunately, this study did not yield statistics on the efficiency of the geometry-induced DW pinning. However, we are confident that the investigated nanowire is representative of the ensemble of fabricated structures. Our interpretation of the measured contrast is supported by our theoretical simulations of the XMCD pattern, which highlight the role of Fresnel diffraction in such investigations [26]. It appears that the biggest issue for the imaging far from the structure lies in the vibrations undergone by the latter due to voltage noise and the field emission in the microscope.

Inducing controlled mechanical oscillations may reduce the influence of these vibrations, but investigating such nanostructures in a horizontal configuration would be easier since no additional device would be needed for imaging. One could think of micromanipulating these onto two laterally separated FEBID-fabricated supports, as a manner of bridge between elevated posts. Not only would this avoid the strong field emission effects that we encountered, it would also allow reduce the structure's vibrations as it could be clamped in place at both its extremities. In this perspective, all the structural engineering described in this report could still be put to use as the structure would be plucked off after its fabrication.

\section{Acknowledgements}

The authors would like to thank Nicolas Rougemaille, Johann Coraux, Olivier Arcizet, Benjamin Pigeau and Jean-Christophe Toussaint for fruitful discussions. This work was supported by Spanish Ministry of Economy and Competitivity through projects No. MAT2014-51982C2-1R, MAT2014-51982C2-2-R and MAT2015-69725-REDT, including FEDER funds, and by the Aragon Regional Government (Construyendo Europa desde Aragón) through project E26, with FEDER funding. Javier PabloNavarro grant is funded by the Ayuda para Contratos Predoctorales para la Formación de Doctores, Convocatoria Res. 05/06/15 (BOE 12/06/15) of the Secretaría de Estado de Investigación, Desarrollo e Innovación in the Subprograma Estatal de Formación of the Spanish Ministry of Economy and Competitiveness (MINECO) with the participation of the European Social Fund. Discussions with Dr. Luis Serrano-Ramón and Dr. Amalio FernándezPacheco about the growth of the Co FEBID nanowires are warmly acknowledged. Michal Staňo acknowledges grant from the Laboratoire d'excellence LANEF in Grenoble (ANR-10-LABX-51-01). 


\section{References}

[1] Kimling J, Kronast F, Martens S, Böhnert T, Martens M, HerreroAlbillos J, Tati-Bismaths L, Merkt U, Nielsch K and Guido M 2011 Physical Review B: Condensed Matter and Materials Physics 84(17) 174406

[2] Elsie Araujo, Armando Encinas, Yenni Velázquez-Galván, Juan Manuel Martínez-Huerta, Gaël Hamoir, Etienne Ferain, and Luc Piraux 2015 Nanoscale 7 1485-1490

[3] Óscar Iglesias-Freire, Cristina Bran, Eider Berganza, Ignacio Mínguez-Bacho, César Magén, Manuel Vázquez and Agustina Asenjo 2015 Nanotechnology 26395702

[4] R Lavín, C Gallardo, JL Palma, J Escrig and JC Denardin 2012 Journal of Magnetism and Magnetic Materials 3242360 - 2362 ISSN 0304-8853

[5] Streubel R, Lee J, Makarov D, Im M Y, Karnaushenko D, Han L, Schäfer R, Fischer P, Kim S K and Schmidt O G 2014 Advanced Materials 26 316-323 ISSN 1521-4095

[6] Mohaddes-Ardabili L, Zheng H, Ogale S B, Hannoyer B, Tian W, Wang J, Lofland S E, Shinde S R, Zhao T, Jia Y, Salamanca-Riba L, Schlom D G, M W and Ramesh R 2004 Nature Materials 3 533-538

[7] Fukunaka Y, Motoyama M, Konishi Y and Ishii R 2006 Electrochemical and Solid-State Letters 9 C62-C64

[8] Li X Z, Wu K L, Ye Y and Wei X W 2014 CrystEngComm 16(21) 4406-4413

[9] Richardson D and Rhen F M 2015 ECS Transactions 64 39-48

[10] Schaefer S, Felix E M, Muench F, Antoni M, Lohaus C, Brotz J, Kunz U, Gartner I and Ensinger W 2016 RSC Advances 6(74) 70033-70039

[11] Zhang H m, Zhang X 1, Zhang J j, Li Z y and Sun H y 2013 Journal of The Electrochemical Society 160 D41-D45

[12] De Teresa J and Fernández-Pacheco A 2014 Applied Physics A 117 1645-1658 ISSN 0947-8396

[13] Teresa J M D, Fernández-Pacheco A, Córdoba R, Serrano-Ramón L, Sangiao S and Ibarra M R 2016 Journal of Physics D: Applied Physics 49243003

[14] Gavagnin M, Wanzenboeck H D, Wachter S, Shawrav M M, Persson A, Gunnarsson K, Svedlindh P, Stöger-Pollach M and Bertagnoll E 2014 Applied Materials and interfaces 6 20254-20260

[15] Fernández-Pacheco A, Serrano-Ramón L, Míchalik J M, Ibarra M R Teresa J M D, O’Brien L, Petit D, Lee J and Cowburn R P 2013 Scientific Reports 31492

[16] Fowlkes J D, Winkler R, Lewis B B, Stanford M G, Plank H and Rack P D 2016 ACS Nano $106163-6172$

[17] Serrano-Ramón L, Córdoba R, Rodríguez L A, Magén C, Snoeck E, Gatel C, Serrano I, Ibarra M R and De Teresa J M 2011 ACS Nano $57781-7787$

[18] Shawrav M M, Taus P, Wanzenboeck H D, Schinnerl M, StögerPollach M, Schwarz S, Steiger-Thirsfeld A and Bertagnolli E 2016 Scientific Reports 634003

[19] Szkudlarek A, Rodrigues Vaz A, Zhang Y, Rudkowski A, Kapusta C, Erni R, Moshkalev S and Utke I 2015 Beilstein Journal of Nanotechnology 6 1508-1517

[20] Fernández-Pacheco A, De Teresa J M, Córdoba R and Ibarra M R 2009 Journal of Physics D: Applied Physics 42055005

[21] Córdoba R, Fernández-Pacheco R, Fernández-Pacheco A, Gloter A, Magén C, Stéphan O, Ibarra M R and De Teresa J M 2011 Nanoscale Research Letters 6592 ISSN 1556-276X

[22] Pablo-Navarro J, Magén C and de Teresa J M 2016 Nanotechnology 27285302

[23] Da Col S, Jamet S, Staňo M, Trapp B, Le Denmat S, Cagnon L, Toussaint J C and Fruchart O 2016 Applied Physics Letters 109 062406

[24] Streubel R, Kravchuk V P, Sheka D D, Makarov D, Kronast F, Schmidt O G and Gaididei Y 2012 Applied Physics Letters 101 132419

[25] Da Col S, Jamet S, Rougemaille N, Locatelli A, Menteş T O, Santos Burgos B, Afid R, Darques M, Cagnon L, Toussaint J C and Fruchart O 2014 Physical Review B 89 180405(R)
[26] Jamet S, Da Col S, Rougemaille N, Wartelle A, Locatelli A, Menteş T O, Santos Burgos B, Afid R, Cagnon L, Fruchart $\mathrm{O}$ and Toussaint J C 2015 Physical Review B 92(14) 144428

[27] Streubel R, Kronast F, Fischer P, Parkinson D, Schmidt O G and Makarov D 2015 Nature Communications 67612

[28] Phatak C, Liu Y, Gulsoy E B, Schmidt D, Franke-Schubert E and Petford-Long A 2014 Nano Letters 14 759-764

[29] Bran C, Berganza E, Palmero E M, Fernandez-Roldan J A, Del Real R P, Aballe L, Foerster M, Asenjo A, Fraile Rodriguez A and Vázquez M 2016 Journal of Materials Chemistry C 4(5) 978-984

[30] Streubel R, Fischer P, Kronast F, Kravchuk V P, Sheka D D, Gaididei Y, Schmidt O G and Makarov D 2016 Journal of Physics D: Applied Physics 49363001

[31] Rouco V, Córdoba R, de Teresa J M, Rodríguez L A, Navau C, DelValle N, Via G, Sánchez A, Monton C, Kronast F, Obradors X, Puig T and Palau A 2017 Scientific Reports 75663

[32] Lubk A, Wolf D, Simon P, Wang C, Sturm S and Felser C 2014 Applied Physics Letters $\mathbf{1 0 5} 173110$

[33] Donnelly C, Guizar-Sicairos M, Scagnoli V, Holler M, Huthwelker T, Menzel A, Vartiainen I, Müller E, Kirk E, Gliga S, Raabe J and Heyderman L J 2015 Physical Review Letters 114(11) 115501

[34] Sanz-Hernández D, Hamans R F, Liao J W, Welbourne A, Lavrijsen $\mathrm{R}$ and Fernández-Pacheco A 2017 Fabrication, detection, and operation of a three-dimensional nanomagnetic conduit arXiv: $1706.03710 \mathrm{v} 1$, accepted for publication in ACS Nano

[35] Belkhou R, Stanescú S, Swaraj S, Besson A, Ledoux M, Hajlaoui M and Dalle D 2015 Journal of Synchrotron Radiation 22 968-979

[36] Belkhou $\mathrm{R}$ et al Room temperature experiments on MnAs (Unpublished)

[37] Begun E, Dobrovolskiy O V, Kompaniiets M, Sachser R, Gspan C, Plank H and Huth M 2015 Nanotechnology 26075301

[38] Lide D 2005 CRC Handbook of Chemistry and Physics, Internet Version (Taylor \& Francis) ISBN 9780849304842

[39] Rohsenow W, Hartnett J and Cho Y 1998 Handbook of heat transfer McGraw-Hill handbooks (McGraw-Hill) ISBN 9780070535558

[40] Wartelle A, Thirion C, Afid R, Jamet S, Col S D, Cagnon L, Toussaint J C, Bachmann J, Bochmann S, Locatelli A, Menteş T O and Fruchart O 2015 IEEE Transactions on Magnetics 51 1-4 ISSN 0018-9464

[41] Krivanek O L, Lovejoy T C, Murfitt M F, Skone G, Batson P E and Dellby N 2014 Journal of Physics: Conference Series $\mathbf{5 2 2} 012023$

[42] Nakajima R, Stöhr J and Idzerda Y U 1999 Physical Review B 59(9) 6421-6429

[43] de Groot F M F, Abbate M, van Elp J, Sawatzky G A, Ma Y J, Chen C T and Sette F 1993 Journal of Physics: Condensed Matter 5 2277

[44] Chi Võ Văn 2013 Epitaxial graphene on metal for new magnetic nanometric systems Phd thesis Université de Grenoble

[45] Córdoba R, Sesé J, Teresa J D and Ibarra M 2010 Microelectronic Engineering 871550 - 1553

[46] Benitez M J, Basith M A, Lamb R J, McGrouther D, McFadzean S, MacLaren D A, Hrabec A, Marrows C H and McVitie S 2015 Physical Review Applied 3(3) 034008

[47] Nahrwold G, Bocklage L, Scholtyssek J M, Matsuyama T, Krüger B, Merkt U and Meier G 2009 Journal of Applied Physics 105 07D511

[48] Glathe S and Mattheis R 2012 Physical Review B 85(2) 024405

[49] Lewis E R, Petit D, Thevenard L, Jausovec A V, O'Brien L, Read D E and Cowburn R P 2009 Applied Physics Letters 95152505

[50] Yershov K V, Kravchuk V P, Sheka D D and Gaididei Y 2015 Physical Review B 92(10) 104412

[51] Lazarus A, Barois T, Perisanu S, Poncharal P, Manneville P, de Langre E, Purcell S T, Vincent P and Ayari A 2010 Applied Physics Letters 96193114

[52] Córdoba R, Lorenzoni M, Pablo-Navarro J, Magén C, Pérez-Murano F and de Teresa J M 2017 manuscript in preparation, submitted to Nanotechnology

[53] Córdoba R, Sharma N, Kölling S, Koenraad P M and Koopmans B 2016 Nanotechnology 27355301

[54] Puydinger dos Santos M V, Velo M F, Domingos R D, Zhang Y, 
Transmission XMCD-PEEM imaging of an engineered vertical FEBID cobalt nanowire

Maeder X, Guerra-Nuñez C, Best J P, Béron F, Pirota K R, Moshkalev S, Diniz J A and Utke I 2016 ACS Applied Materials \& Interfaces 8 32496-32503

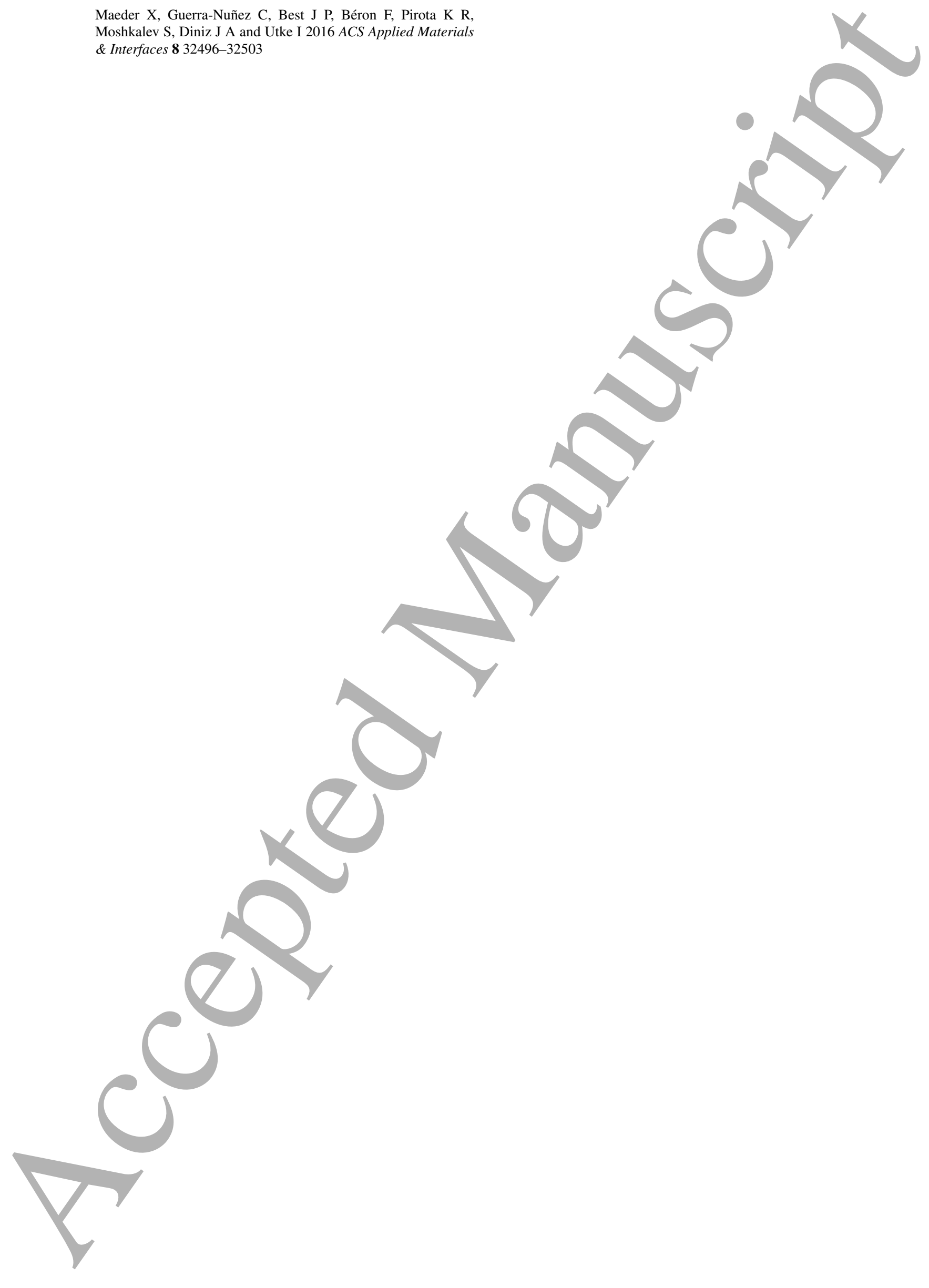

